\title{
Building Half-Metallicity in Graphene Nanoribbons by Direct Control over Edge States Occupation
}

\author{
X. H. Zheng, ${ }^{\dagger}$ X. L. Wang, ${ }^{\dagger}$ T. A. Abtew ${ }^{\ddagger}$ and Z. Zeng ${ }^{*}, \dagger$ \\ Key Laboratory of Materials Physics, Institute of Solid State Physics, Chinese Academy of Sciences, Hefei \\ 230031, China, and Department of Physics, University at Buffalo, State University of New York, \\ Buffalo, New York 14260
}

Received: November 25, 2009; Revised Manuscript Received: January 25, 2010

\begin{abstract}
Electronic structures of zigzag edged graphene nanoribbons (ZGNRs) doped with boron (B) or nitrogen (N) atoms are investigated by spin polarized first-principles calculations. We find that ZGNRs can be tuned to be either semiconducting, half-metallic, or metallic by controlling the distance of the impurity atoms to the edges. A new scheme is identified to achieve full half-metallicity in ZGNRs by doping B atom at one edge and $\mathrm{N}$ atom at the other. We find that the origin of the half-metallicity is due to interaction between the edge states and $\mathrm{B} / \mathrm{N}$ atoms which results in direct control over the electron occupation of the edge states. This mechanism is so robust that full half-metallicity can always be produced in ZGNRs irrespective of the ribbon width, which opens new possibilities for applications of ZGNRs in spintronic devices.
\end{abstract}

\section{Introduction}

Half-metallicity has long been at the center of research since its first discovery ${ }^{1}$ in 1983 and has been observed in many materials, such as Heusler compounds, ${ }^{1}$ manganese perovskites, ${ }^{2}$ organometallic benzene-vanadium wire, ${ }^{3}$ and metal-DNA complexes, ${ }^{4}$ etc. The importance of half-metallicity lies in the coexistence of the metallic nature of electrons with one spin orientation and an insulating nature for the other spin orientation. This results in a completely spin polarized current in materials with half-metallicity. Such materials are called half-metals and are promising candidates for spintronics applications. Recently, graphene, a strictly two-dimensional flat single layer of graphite, has received extensive attention due to its remarkable structural and electronic properties. ${ }^{5,6}$ For the purposes of device applications, it can be made into quasi-one-dimensional nanoribbons either by cutting mechanically exfoliated graphenes and patterning by lithographic techniques ${ }^{7,8}$ or by tuning the epitaxial growth of graphenes. ${ }^{9,10}$ Two categories of nanoribbons receive the most interest: one with armchair edges and the other with zigzag edges. Graphene nanoribbons with different edge shapes (armchair or zigzag) or ribbon widths show different conductivity (semiconducting or metallic). Furthermore, the semiconducting ribbons with different widths have different band gaps that provide various kinds of building blocks needed in nanoelectronic devices. Especially, due to the local magnetism on the edge carbon atoms, the zigzag edged graphene nanoribbons (ZGNRs) may be very useful for realizing spin polarized transport and thus for applications in spintronic devices.

Interestingly, not long ago, half-metallicity was predicted in graphene nanoribbons by Son et al. ${ }^{11}$ They proposed that by applying a transverse electrical field across a ZGNR, halfmetallicity can appear as a result of opposite shift in energy of the spin-degenerate localized edge states on the two sides. However, Kan et al. demonstrated that a very high critical

* To whom correspondence should be addressed. E-mail: zzeng@ theory.issp.ac.cn.

${ }^{\dagger}$ Chinese Academy of Sciences.

* University at Buffalo, State University of New York. electrical field is required to achieve half-metallicity in ZGNRs. ${ }^{12}$ Alternatively, they proposed a chemical decoration technique to realize half-metallicity by replacing the terminating hydrogen $(\mathrm{H})$ atoms on one edge by $\mathrm{NO}_{2}$ groups while replacing those on the other edge by $\mathrm{CH}_{3}$ groups. ${ }^{13}$ The different effective potentials induced by these functional groups shift the energy of the edge states on the two sides in an opposite direction that brings the half-metallicity. In another work of Kan et al., a scheme of replacing periodically an armchair carbon chain across the ZGNR by a boron-nitrogen (BN) chain is suggested to produce half-metallicity. ${ }^{14}$ Very recently, Dutta et al. proposed a scheme of replacing the middle zigzag carbon chains along the nanoribbon by zigzag BN chains to obtain half-metallicity. ${ }^{15}$

In this work, in contrast to the large scale chain substitution schemes proposed by Kan or Dutta, we provide a new way of producing half-metallicity by chemical doping in which only very few carbon atoms are replaced by impurity atoms. It is well-known that in ZGNRs all the atoms inside one edge are aligned ferromagnetically, but the atoms between the two edges are antiferromagtically coupled and the band structures for one spin channel $(\alpha)$ and the other spin channel $(\beta)$ are completely degenerate. ${ }^{11,16-18}$ This results in a zero magnetic moment and a nonspin polarized transport. Such spin degeneracy should be broken in order to realize spin polarized transport through ZGNRs. All the magnetism related or spin polarized properties in ZGNRs are directly related to the edge states which are localized at the edges. Therefore, a natural way to break the spin degeneracy is to tailor the edge states by various ways, ${ }^{11,13}$ for example, by chemical doping. The most frequently used dopants (B or N) in carbon systems ${ }^{14,15,19-22}$ are adopted in our work to probe the doping effects of various doping situations. Doping B or N atoms strongly affects the electronic structures of ZGNRs and tunes ZGNRs to be either metallic, semiconducting, or nearly half-metallic. Furthermore, full half-metallicity can be achieved by doping $\mathrm{B}$ atoms in one edge and $\mathrm{N}$ atoms in the other.

The rest of this paper is organized as follows: in section 2, we give a brief description of the geometry used for the ZGNRs and of the computational method, while in section 3 the main 


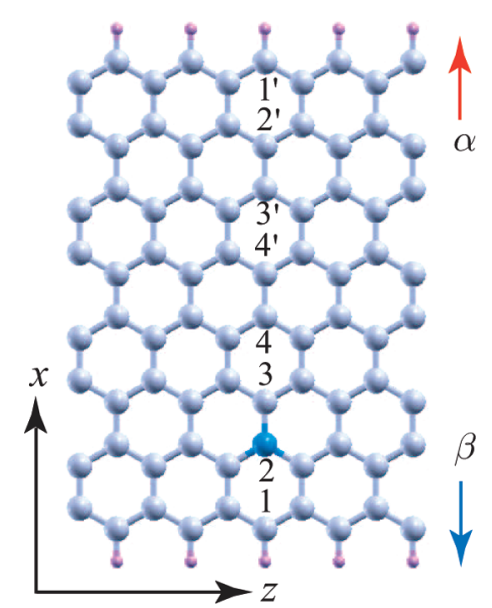

Figure 1. Supercell of a hydrogen-passivated 8-ZGNR in which five unit cells along the ribbon are included. The numbers 1, 2, 3, 4 and $1^{\prime}$, $2^{\prime}, 3^{\prime}, 4^{\prime}$ counting from different edges indicate the doping sites. The atom shown in blue is one of the doping sites and just for a guide to the eye. $\alpha$ and $\beta$ indices represent antiferromagnetically aligned spins.

results are presented and discussed. Finally, in section 4 we draw conclusions.

\section{Computational Details}

The doping effects in ZGNRs are analyzed by performing density functional theory calculations using SIESTA code which employs norm-conserving pseudopotentials and linear combinations of atomic orbitals as basis sets. ${ }^{23}$ The wave function is expanded with a double- $\zeta$ polarized (DZP) basis set, and the exchange-correlation potential is treated at the level of generalized gradient approximation (GGA), with the form of PerdewBurke-Ernzerhof (PBE). ${ }^{24}$ The fineness of real space grid is determined by an equivalent plane wave cutoff 200 Ryd. In this work, we consider chemically doped ZGNRs with various widths and start with hydrogen passivated 8-ZGNR (eight zigzag carbon chains along the $z$ axis, the periodic direction). The supercell in our calculations is chosen to contain five armchair rows or unit cells with length of $12.3 \AA$ along the ribbon (see Figure 1). The lattice vectors along the $x$ and $y$ axis are set to be $43 \AA$ and $16 \AA$, respectively, which include a vacuum big enough to avoid interactions from the adjacent neighbors. The Brillouin zone is sampled by a $1 \times 1 \times 20 k$-point grid. We begin with doping only one impurity atom in the supercell and systematically investigate the doping effect on the electronic structures of the ZGNRs as a function of the distance from the impurity atom to the ribbon edges. Specifically, the carbon atoms at positions $1,2,3$, and 4 are replaced by a $\mathrm{B}$ or $\mathrm{N}$ atom each time. Due to the spin symmetry of the ribbon, the doping effects at $1^{\prime}, 2^{\prime}, 3^{\prime}$, and $4^{\prime}$ can be obtained from the results of the previous schemes of doping at sites $1,2,3$, and 4 by exchanging the two spin indices. All the structures are fully relaxed to reach the force tolerance of $0.01 \mathrm{eV} / \AA$. Both ferromagnetic (FM) and antiferromagnetic (AFM) initial configurations of the atoms between the two edges are considered.

\section{Results and Discussion}

Since in the ground state of pristine ZGNRs, the edge states at the two sides are antiferromagnetically coupled, at first, only antiferromagnetic (AFM) initial configurations of the atoms between the two edges of all the structures are considered. After full relaxation, all the doped structures are still in AFM
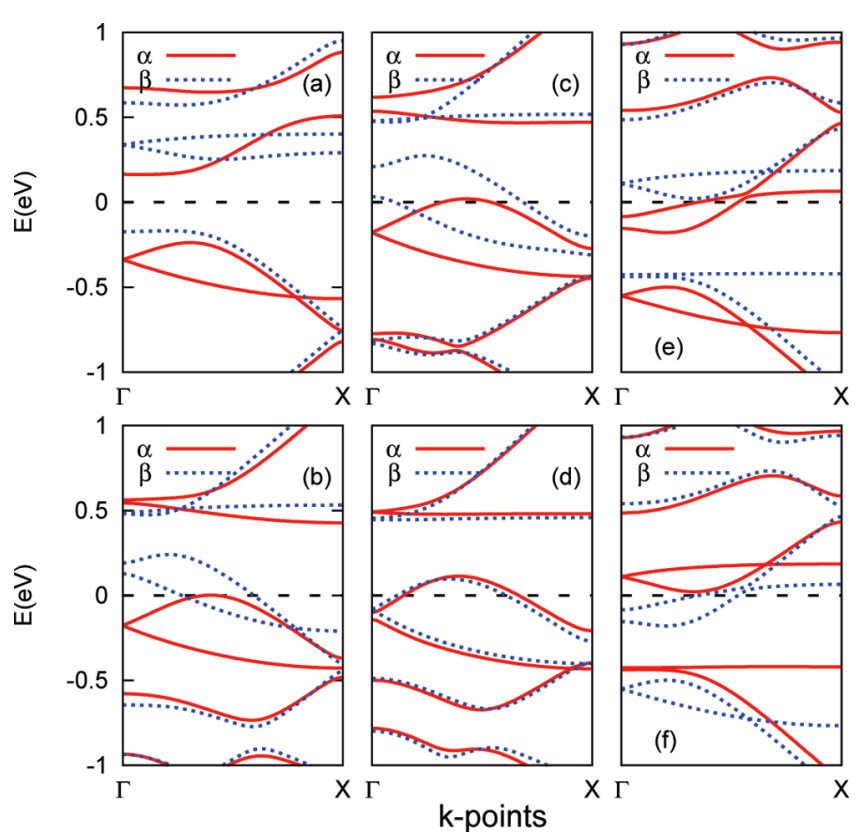

Figure 2. Band structures of ZGNR with dopant B at (a) site 1; (b) site 2; (c) site 3; (d) site 4 and with dopant $\mathrm{N}$ at (e) site 2; (f) site $2^{\prime}$. The Fermi level is set to $0 \mathrm{eV}$ throughout the paper.

configuration. However, significant changes in the electronic structure are obtained after B is doped. Degeneracy of both the conduction bands and the valence bands is broken due to the existence of the B atom. The conductivity of the doped ribbon is highly dependent on the doping site as can be seen from the band structures. When one edge carbon (at site 1; see Figure 1) is substituted by a B atom, the ribbon is semiconducting (see Figure 2a). On the other hand, it is metallic when the B atom is doped in the middle at site 4 (see Figure $2 \mathrm{~d}$ ). When the B atom is doped at site 2 or 3 , the ribbon turns to be nearly halfmetallic with only the two valence bands of the $\beta$ spin channel crossing the Fermi level (see Figure $2 b$ and $c$ ). We define it as "nearly half-metallic" since the valence bands of the $\alpha$ channel are right below and very close to the Fermi level. At finite temperature, electrons in these bands will also take part in conduction. The influence of $\mathrm{N}$ doping is similar to the one for $\mathrm{B}$ doping. The main difference is that the bands crossing the Fermi level are the conduction bands in the $\alpha$ spin channel when the $\mathrm{N}$ atom is doped at site 2 or 3 (see Figure 2e).

In order to understand why it is the $\beta$ spin channel that is conducting in the B doping case while it is the $\alpha$ spin channel that is conducting in the $\mathrm{N}$ doping case, we have investigated the nature of the edge states in the valence bands and conduction bands (see Figure 3). It can be seen that in ZGNRs, the wave functions with $\alpha$ spin in the valence bands are localized at the top edge (see Figure $3(v-\alpha)$ ) while those with $\beta$ spin are localized at the bottom edge (see Figure $3(v-\beta)$ ). On the contrary, in the conduction bands, the wave functions with $\alpha$ spin are localized at the bottom edge (see Figure 3(c- $-\alpha)$ ) while those with $\beta$ spin are localized at the top edge (see Figure $3(c-\beta))$. Particularly, it is clearly shown that the magnitude of the edge states at the $\gamma$ point extends over only the first three zigzag chains from the edges and contribution from all the other atoms to these states is negligible. Based on this, a physical picture can be given to understand the big difference in the effects of B and $\mathrm{N}$ doping. When one $\mathrm{B}$ atom is doped at site 2 or 3 (see Figure $3(\nu-\alpha)$ and $(v-\beta)$ ), it introduces a hole and the Fermi level shifts down to the valence bands. Since the B atom is close to the bottom edge and far from the top edge, it 

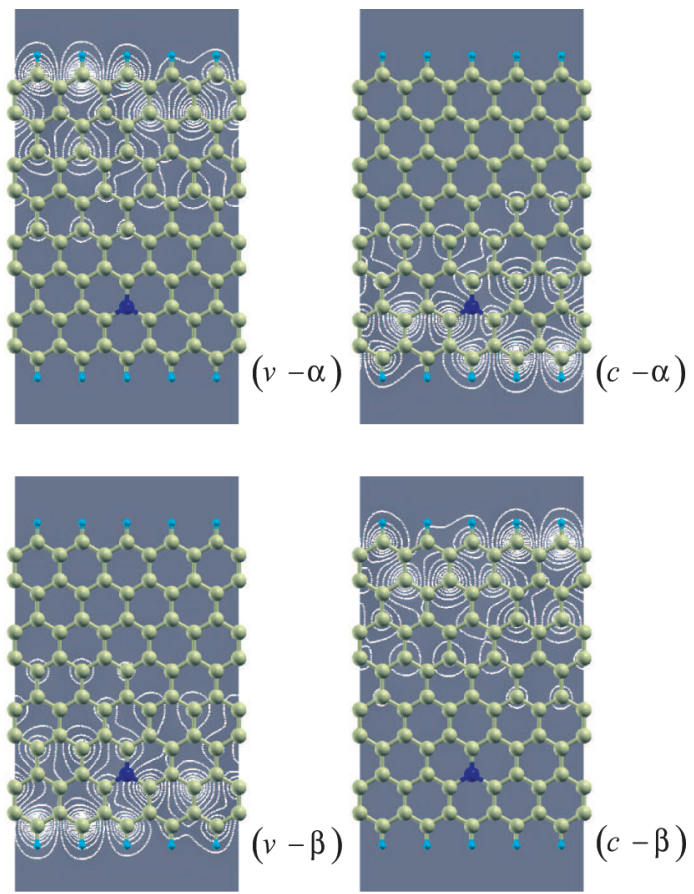

Figure 3. Contour of wave functions (edge states) of the valence bands and conduction bands at the $\Gamma$ point for the undoped 8-ZGNR shown in Figure 1. $v$ means valence bands, while c means conduction bands. $\alpha$ and $\beta$ are the spin indices. The carbon atom shown in blue indicates one of the possible doping sites, namely, doping site 2 .

interacts with the edge states with $\beta$ spin localized at the bottom edge and attracts electrons from them. Thus, the $\beta$ spin channel becomes partially filled while keeping the $\alpha$ spin channel still fully occupied (see Figure $2 \mathrm{~b}$ and $\mathrm{c}$ ). However, when one $\mathrm{N}$ atom is doped at site 2 or 3 (see Figure $3(c-\alpha)$ and $(c-\beta)$ ), it introduces an electron and the Fermi level shifts up to the conduction bands. This excess electron transfers to the $\alpha$ channel since the edge states of this channel are located at the bottom edge. This makes the $\alpha$ channel in the conduction bands partially filled and the $\beta$ channel still empty (see Figure 2e).

With only $\mathrm{B}$ doping or $\mathrm{N}$ doping at site 2 or 3 at the bottom edge, although we have the bands of only one spin channel conducting, the bands from the other spin channel are also very close to the Fermi level. In order to get good half-metallicity, we need to push away this spin channel from the Fermi level to make it an insulator. This can be achieved by introducing electrons (e.g., by doping one more $\mathrm{N}$ atom) to the $\mathrm{B}$ doped system to shift up the Fermi level or by introducing holes (e.g., by doping one more $\mathrm{B}$ atom) to the $\mathrm{N}$ doped system to shift down the Fermi level. The question is, where to dope the second atom? It is important and natural to note that if the doping site of the $\mathrm{N}$ doped system is at $2^{\prime}$ or $3^{\prime}$ in the top edge, then the conducting bands will be from the $\beta$ spin channel (see Figure $2 f)$. Subsequently, if the doping site of the B doped system is at 2 or 3 and the doping site of the $\mathrm{N}$ doped system is at $2^{\prime}$ or $3^{\prime}$, then the same spin channel, namely, the $\beta$ channel, is conducting in both cases. If two carbon atoms on opposite sides (one at 2 or 3 and the other at $2^{\prime}$ or $3^{\prime}$ ) are replaced by a B and a $\mathrm{N}$ atom, the demands for electron or hole in both the $\mathrm{B}$ doping and the $\mathrm{N}$ doping schemes to shift the Fermi level can be satisfied. Moreover, the combination of these two schemes will promote each other. Thus, a full half-metallicity can be achieved as shown in our calculations (see Figure 4a). It should be noted that the impurity atoms can lie at any site in the rows where site $2,3,2^{\prime}$, or $3^{\prime}$ is located along the $z$ direction.

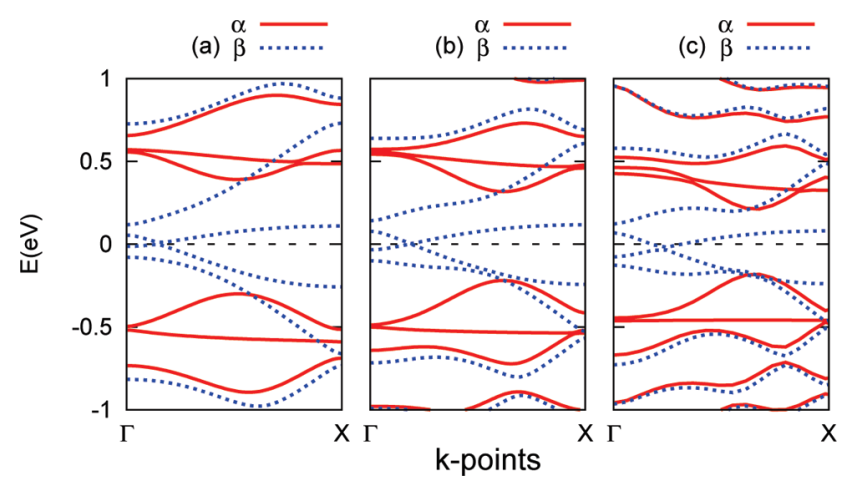

Figure 4. Band structures with one $\mathrm{B}$ and one $\mathrm{N}$ atom doped at different edges for (a) 8-ZGNR; (b) 12-ZGNR; (c) 18-ZGNR. The doping sites for the $\mathrm{B}$ and $\mathrm{N}$ atoms are both at the second carbon atom from the different edges.

In order to investigate the robustness of this $\mathrm{B}-\mathrm{N}$ pair doping scheme for producing half-metallicity in ZGNRs with different ribbon widths, calculations with varying ZGNR width (8-ZGNR, 12-ZGNR, and 18-ZGNR) are performed. Similar observations are obtained in all the cases. In each case, when B doping is at site 2 or 3 at one edge and $\mathrm{N}$ doping is at site $2^{\prime}$ or $3^{\prime}$ at the other edge, half-metallicity can always be observed, irrespective of the ribbon width. Figures $4 \mathrm{~b}$ and $\mathrm{c}$ show the band structures for the 12-ZGNR and 18-ZGRN with one $\mathrm{B}$ and $\mathrm{N}$ atom doped at sites 2 and $2^{\prime}$ at the different edges. The only difference is that the band gap of the insulating channel decreases inversely with the increase of the ribbon width (compare the three cases in Figure 4).

Furthermore, the effect of doping concentration has also been investigated by choosing supercells with different sizes. It is found that doping concentration is important for obtaining halfmetallicity. We define the doping ratio as

$$
p=1: N_{\mathrm{u}}
$$

where 1 stands for doping one $\mathrm{B}-\mathrm{N}$ pair in one supercell and $N_{\mathrm{u}}$ is the number of unit cells along the $z$ direction. Our calculations show that half-metallicity appears only with the doping ratio $1: 7 \leq p \leq 1: 3$. For both $p=1: 1$ and $p=1: 2$ cases, the system has a nonmagnetic ground state (see Figure $5 \mathrm{a}$ and $\mathrm{b}$ ). When $p=1: 3$ (see Figure $5 \mathrm{c}$ ), the system begins to show half-metallicity. However, with $p \leq 1: 8$, the system turns to be a semiconductor, with an energy gap of one spin channel much smaller than that of the other spin channel (see Figure 5d). The effects of the doping become weaker and weaker as the doping concentration decreases. The valence bands and the conduction bands get separated, and thus an energy gap appears. In the limit of $N_{\mathrm{u}} \rightarrow \infty$, the electronic structure of the doped ZGNRs will converge to that of the pristine ZGNRs. Although a small gap opens for the conducting channel when the doping concentration decreases to some extent, it is still of great importance since in certain energy ranges around the Fermi level, one spin channel conducts while the other does not (see the energy range $[-0.32,0.38] \mathrm{eV}$ in Figure $5 \mathrm{~d}$ ).

Thus far, only the best situation with doping $\mathrm{B}$ and $\mathrm{N}$ atoms at different edges has been discussed. In fact, we have considered the random doping situations. Since the second atom $\mathrm{N}$ (or B) is mainly used to provide an electron (or hole) to shift the Fermi level, theoretically, it can lie at any position as long as it does not affect the same edge states simulta- 
(a) $\stackrel{\alpha}{\beta} \overline{\ldots \ldots}$

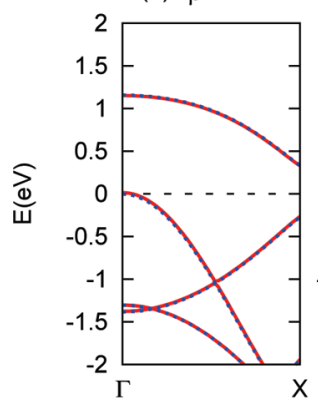

(b) $\alpha \underset{\beta}{\beta \ldots . . .}$

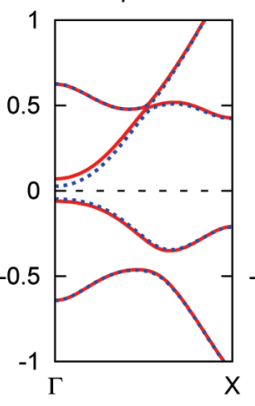

(c) $\stackrel{\alpha}{\beta} \overline{\ldots \ldots}$

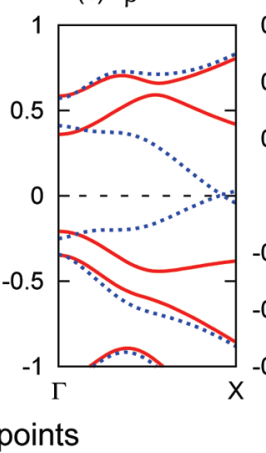

(d) $\stackrel{\alpha}{\beta}$

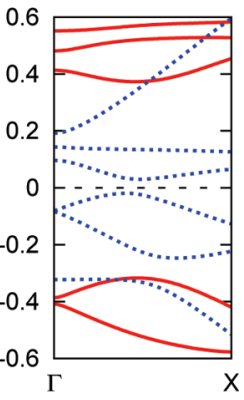

Figure 5. Band structures for $B N$ doped 8 -ZGNR with doping ratio $p$ as (a) $1: 1$; (b) $1: 2$; (c) 1:3; (d) $1: 8$.

neously with the first impurity atom B (or N). Our calculations with random doping show that even if the second atom is in the inner rows of the ribbon, half-metallicity can still be observed. Nevertheless, for some doping positions, a minigap may exist for the conducting channel (not shown). But we can still see that the band gap of one spin channel is much bigger than that of the other channel, much like in the case shown in Figure 5d.

Finally, to investigate the relative stability of different magnetic configurations, we have done calculations for other spin configurations, namely, ferromagnetic (FM) order between the edges and nonmagnetic (NM) situation, for the cases in which half-metallicity is predicted. From these calculations, we find that AFM is the ground state for all the cases. In particular, for the case in Figure $4 \mathrm{a}$, the energy difference between the $\mathrm{FM}$ and AFM is $11 \mathrm{meV}$. On the other hand, the energy difference between $\mathrm{NM}$ and AFM is $186 \mathrm{meV}$, suggesting that AFM is the most stable state and half-metallicity can be achieved in ZGNRs by the proposed $\mathrm{B}-\mathrm{N}$ doping scheme.

\section{Conclusion}

In conclusion, we have investigated the $\mathrm{B}$ and $\mathrm{N}$ doping effects on the electronic structure of ZGNRs using firstprinciples calculations. It is found that doping $\mathrm{B}$ or $\mathrm{N}$ atoms affects the localized edge states on the two sides differently, which significantly changes the conductivity of the ZGNRs. Depending on the doping sites, ZGNRs can be tuned to be either semiconductors, metals, or nearly half-metals. Our calculations suggest that, by jointly applying electron $(\mathrm{N})$ doping and hole (B) doping at different parts/edges of the ribbon, we can directly control the electron occupation of specific edge states and halfmetallicity can always be produced irrespective of the ribbon width. This fact provides a new scheme for building halfmetallicity in ZGNRs and opens new possibilities of applications of ZGNRs in spintronics.

Acknowledgment. This work was supported by the National Science Foundation of China under grant nos. 10774148 and 10904148, the Special Funds for Major State Basic Research Project of China(973) under grant no. 2007CB925004, 863 Project, Knowledge Innovation Program of Chinese Academy of Sciences, and Director Grants of CASHIPS. Part of the calculations were performed in the Center for
Computational Science of CASHIPS and the Shanghai Supercomputer Center.

\section{References and Notes}

(1) de Groot, R. A.; Mueller, F. M.; Engen, P. G. v.; Buschow, K. H. J. Phys. Rev. Lett. 1983, 50, 2024-2027.

(2) Park, J.-H.; Vescovo, E.; Kim, H.-J.; Kwon, C.; Ramesh, R.; Venkatesan, T. Nature (London, U.K.) 1998, 392, 794.

(3) Maslyuk, V. V.; Bagrets, A.; Meded, V.; Arnold, A.; Evers, F.; Brandbyge, M.; Bredow, T.; Mertig, I. Phys. Rev. Lett. 2006, 97, 097201.

(4) Mallajosyula, S. S.; Pati, S. K. J. Phys. Chem. B 2007, 111, 13877.

(5) Novoselov, K. S.; Geim, A. K.; Morozov, S. V.; Jiang, D.; Zhang, Y.; Dubonos, S. V.; Grigorieva, I. V.; Firsov, A. A. Science 2004, 306, 666.

(6) Geim, A. K.; Novoselov, K. S. Nat. Mater. 2007, 6, 183.

(7) Tapaszto, L.; Dobrik, G.; Lambin, P.; Biro, L. P. Nat. Nanotechnol. 2008, 3, 397.

(8) Datta, S. S.; Strachan, D. R.; Khamis, S. M.; Charlie-Johnson, A. T. Nano Lett. 2008, 8, 1912.

(9) Berger, C.; Song, Z.; Li, T.; Li, X.; Ogbazghi, A. Y.; Feng, R.; Dai, Z.; Marchenkov, A. N.; Conrad, E. H.; First, P. N.; de Heer, W. A. J. Phys. Chem. B 2004, 108, 19912.

(10) Berger, C.; Song, Z.; Li, X.; Wu, X.; Brown, N.; Naud, C.; Mayou, D.; Li, T.; Hass, J.; Marchenkov, A. N.; Conrad, E. H.; First, P. N.; de Heer, W. A. Science 2006, 312, 1191.

(11) Son, Y. W.; Cohen, M. L.; Louie, S. G. Nature (London, U.K.) 2006, 444, 347.

(12) Kan, E.-J.; Li, Z. Y.; Yang, J. L.; Hou, J. G. Appl. Phys. Lett. 2007, $91,243116$.

(13) Kan, E.-J.; Li, Z. Y.; Yang, J. L.; Hou, J. G. J. Am. Chem. Soc. 2008, 130, 4224.

(14) Kan, E.-J.; Wu, X. J.; Li, Z. Y.; Zeng, X. C.; Yang, J. L.; Hou, J. G. J. Chem. Phys. 2008, 129, 084712.

(15) Dutta, S.; Manna, A. K.; Pati, S. K. Phys. Rev. Lett. 2009, 102, 096601.

(16) Fujita, M.; Wakabayashi, K.; Nakada, K.; Kusakabe, K. J. Phys. Soc. Jpn. 1996, 65, 1920.

(17) Okada, S.; Oshiyama, A. Phys. Rev. Lett. 2001, 87, 146803.

(18) Lee, H.; Son, Y.-W.; Park, N.; Han, S.; Yu, J. Phys. Rev. B 2005, 72,174431 .

(19) Martins, T. B.; Miwa, R. H.; da Silva, A. J. R.; Fazzio, A. Phys. Rev. Lett. 2007, 98, 196803.

(20) Son, Y.-W.; Ihm, J.; Cohen, M. L.; Louie, S. G.; Choi, H. J. Phys. Rev. Lett. 2005, 95, 216602.

(21) Biel, B.; Blase, X.; Triozon, F. m. c.; Roche, S. Phys. Rev. Lett. 2009, 102, 096803.

(22) Zheng, X. H.; Rungger, I.; Zeng, Z.; Sanvito, S. Phys. Rev. B 2009, $80,235426$.

(23) Soler, J. M.; Artacho, E.; Gale, J. D.; Garcia, A.; Junquera, J.; Ordejon, P.; Sanchez-Portal, D. J. Phys.: Condens. Matter 2002, 14, 2745.

(24) Burke, K.; Perdew, J. P.; Ernzerhof, M. Int. J. Quantum Chem. 1997, 61, 287.

JP911203N 